\title{
Quasi-free limit in the deuteron-deuteron three-body break-up process
}

R. Ramazani-Sharifabadi ${ }^{1,2 \star}{ }^{2}$, H. R. Amir-Ahmadi ${ }^{1}$, M. T. Bayat ${ }^{1}$, I. Ciepat ${ }^{3}$, M. Eslami-Kalantari ${ }^{4}$, N. Kalantar-Nayestanaki ${ }^{1}$, St. Kistryn ${ }^{5}$, A. Kozela ${ }^{6}$, M. Mahjour-Shafiei ${ }^{2 \star}$, H. Mardanpour ${ }^{1 \star}$, J. G. Messchendorp ${ }^{1 \star}$, M. Mohammadi-Dadkan ${ }^{1,7}$,

A. Ramazani-Moghaddam-Arani ${ }^{8}$, E. Stephan ${ }^{9}$, and H. Tavakoli-Zaniani ${ }^{1,4}$

1 KVI-CART, University of Groningen, Groningen, The Netherlands

2 Department of Physics, University of Tehran, Tehran, Iran

3 Institute of Nuclear Physics, PAS, PL-31342, Kraków, Poland

4 Department of Physics, School of Science, Yazd University, Yazd, Iran

5 Institute of Physics, Jagiellonian University, Kraków, Poland

6 Institute of Nuclear Physics, Polish Academy of Sciences, Kraków, Poland

7 Department of Physics, University of Sistan and Baluchestan, Zahedan, Iran

8 Department of Physics, Faculty of Science, University of Kashan, Kashan, Iran

9 Institute of Physics, University of Silesia, Chorzow, Poland

* r.ramazani.sharifabadi@rug.nl * mmshafiei@ut.ac.ir * j.g.messchendorp@rug.nl

March 27, 2020

Proceedings for the 24th edition of European Few Body Conference, Surrey, UK, 2-4 September 2019

\begin{abstract}
Detailed measurements of vector and tensor analyzing powers of the ${ }^{2} \mathrm{H}(\vec{d}, d p) n$ breakup process are presented. The data were obtained using a polarized deuteron-beam with an energy of $65 \mathrm{MeV} /$ nucleon impinging on a liquid-deuterium target. The experiment was conducted at the AGOR facility at KVI using the BINA $4 \pi$-detection system. The focus of this contribution is to analyze data of the $d d$ scattering process in the regime at which the neutron acts as a spectator, which we refer to as the quasi-free (QF) limit. To achieve this, events for which the final-state deuteron and proton are coplanar have been analyzed and the data have been sorted for various reconstructed momenta of the missing neutron. In the limit of vanishing neutron momentum and at small deuteron-proton momentum transfer, the data match very well with measured and predicted spin observables of the elastic deuteron-proton scattering process. The agreement deteriorates rapidly with increasing neutron momentum and deuteron-proton momentum transfer. The results of coplanar configurations in four-body phase space are compared with the results of recent available theoretical calculations based on the Single-Scattering Approximation.
\end{abstract}

\section{Contents}

1 Introduction 2

2 Experimental setup $\quad 2$ 


\section{Introduction}

Development of boson-exchange models [1] led to several phenomenological nucleon-nucleon (NN) potentials which have successfully described the interaction between two nucleons with $\chi^{2} \sim 1$. However, applying these high-precision NN potentials to describe systems composed of at least three nucleons shows striking discrepancies between theoretical calculations and experimental observables [2]. The inclusion of the three-nucleon force (3NF) effects generally improves the agreement with the data [3].

Presently, an extensive database is available for the nucleon-deuteron scattering process at different energies below pion-production threshold [4-18]. Contrary to this, the experimental database for $4 \mathrm{~N}$ systems in the low-energy regime below three- and four-body break-up threshold is limited [19]. At these low energies, the calculations are very reliable, but the effect of $3 \mathrm{NF}$ is very small and hard to measure. Above the break-up thresholds and below the pion-production threshold, hence, at intermediate energies, the $4 \mathrm{~N}$ database becomes even more scarce [20]. Exact theoretical calculations for four nucleon systems are still limited to the energy ranges below $30 \mathrm{MeV}$ [21-28]. At intermediate energies, there are no ab-initio calculations. For this region, data can be compared to calculations that are based on some approximations [20]. Recent theoretical calculations are available that are based on the singlescattering approximation (SSA). These calculations are expected to be valid in the quasi-free (QF) regions for beam energies larger than $100 \mathrm{MeV}[29,30]$.

In this paper, we present the results of an investigation of the ${ }^{2} \mathrm{H}(\vec{d}, d p) n$ break-up process for coplanar configurations including Quasi-Free (QF) region at deuteron-beam energy of $65 \mathrm{MeV} /$ nucleon. The data were obtained by making use of a vector- and tensor-polarized deuteron beam that was provided by the AGOR facility at KVI in Groningen, the Netherlands. The QF domain is investigated via a detailed analysis of the three-body break-up reaction. The results are compared with the results of recent theoretical calculations based on SSA. Through these comparisons, the validity of SSA approximation is investigated in the QF regions.

\section{Experimental setup}

The experimental setup involved in $d d$ scattering process is the BINA, Big-Instrument for Nuclear-Polarization Analysis. Vector- and tensor-polarized deuteron beams were produced by the atomic Polarized Ion Source (POLIS) with nominal polarization values between 60 $80 \%$ of the maximum theoretical values [31]. The deuteron beam was accelerated up to 130 $\mathrm{MeV}$ by the superconducting AGOR facility. The accelerated deuteron beam impinged on the liquid deuterium target [32] inside the scattering chamber of BINA during around 51 hours of beam time. The thickness of the target cell was $3.85 \mathrm{~mm}$ with an uncertainty of $5 \%$. BINA was developed with nearly full coverage of the geometrical acceptance, and the detector provides a measurement of the energy and scattering angles of final-state protons and deuterons with the capability for particle identification $[14,33,34]$. 
The polarization of the deuteron beam was monitored with a Lamb-Shift Polarimeter [35] at the low-energy beam line and determined with BINA after the beam acceleration using a measurement of the elastic deuteron-proton scattering. The vector and tensor polarizations of the deuteron beams were found to be $p_{Z}=-0.601 \pm 0.029$ and $p_{Z Z}=-1.517 \pm 0.032$, respectively, whereby the errors include uncertainties in the analyzing powers in elastic $d p$ scattering. The polarization of the deuteron beam was monitored for different periods of experiment and found to be stable within statistical uncertainties [36].

\section{Event selection and analysis method}

Spin observables of the break-up channel have been measured in a nearly-background-free experiment. The identification of the three-body break-up channel from other hadronic channels has been done using the measured energies, scattering angles, and time-of-flight (TOF) of the detected particles. The hardware trigger was biased on the selection of events for which both the proton and deuteron were registered in coincidence by the forward wall of BINA corresponding to small scattering angles.

The prerequisites of this analysis on the three-body break-up channel is the energy calibration of the ten $E$ scintillator bars in the forward wall of the BINA and the particle identification. Two methods of energy calibration procedure have been exploited to investigate the sensitivity of the final results to the energy calibration procedures. For the first calibration method, we followed the procedure given in Ref. [34]. In this method, the detector response has been parametrized by a three-parameter fit-function. In the second method, we increased the number of degrees-of-freedom in the fit to five parameters for the calibration function to match the experimental data points to the theoretical $S$-curve.

Figure 1 shows the energy correlation between deuteron and proton ( $S$-curve) for the configuration $\left(\theta_{d}, \theta_{p}, \phi_{12}\right)=\left(25^{\circ} \pm 1,25^{\circ} \pm 1,180^{\circ} \pm 5\right)$ after energy calibration and particle identification. Here, $\phi_{12}$ is the difference of the azimuthal angles of the two particles. The solid line represents the expected kinematical $S$-curve. The tail on below the $S$-curve is dominantly those events under hadronic interaction. The $S$-curve is divided into $S$-bins with a width of $10 \mathrm{MeV}$. One of those bins are indicated by the dashed lines and used to count the number of events inside the $S$-bin. To count the number of break-up events in the interval of $S-\frac{\Delta S}{2}$ and $S+\frac{\Delta S}{2}$, the events are projected on a line perpendicular to the $S$-curve for each $S$-bin with The value of $\Delta S$ of $\pm 5 \mathrm{MeV}$. The result of projected events for a particular $S$-bin is presented in the inset of Fig. 1. This spectrum consists of a peak around zero belonging to the break-up events with a negligible amount of accidental background. The peak shows that the majority of break-up events deposit all their energies in the scintillator. The tail on the left-hand side of the peak corresponds again to events which undergo hadronic interactions inside the scintillator. The solid line in the inset of Fig. 1 is the result of a fit through the data based on a Gaussian-distributed signal combined with a third-order polynomial representing the contribution of the hadronic interactions. The number of events under the peak is used for further analysis.

The interaction of a polarized beam with an unpolarized target produces an azimuthal asymmetry in the cross section. This asymmetry is proportional to the product of the magnitude of the polarization and another observable, namely analyzing power. Vector- and tensorpolarized beams give the possibility to measure various analyzing powers by studying the azimuthal asymmetry in the differential cross section. The cross section of any reaction with 


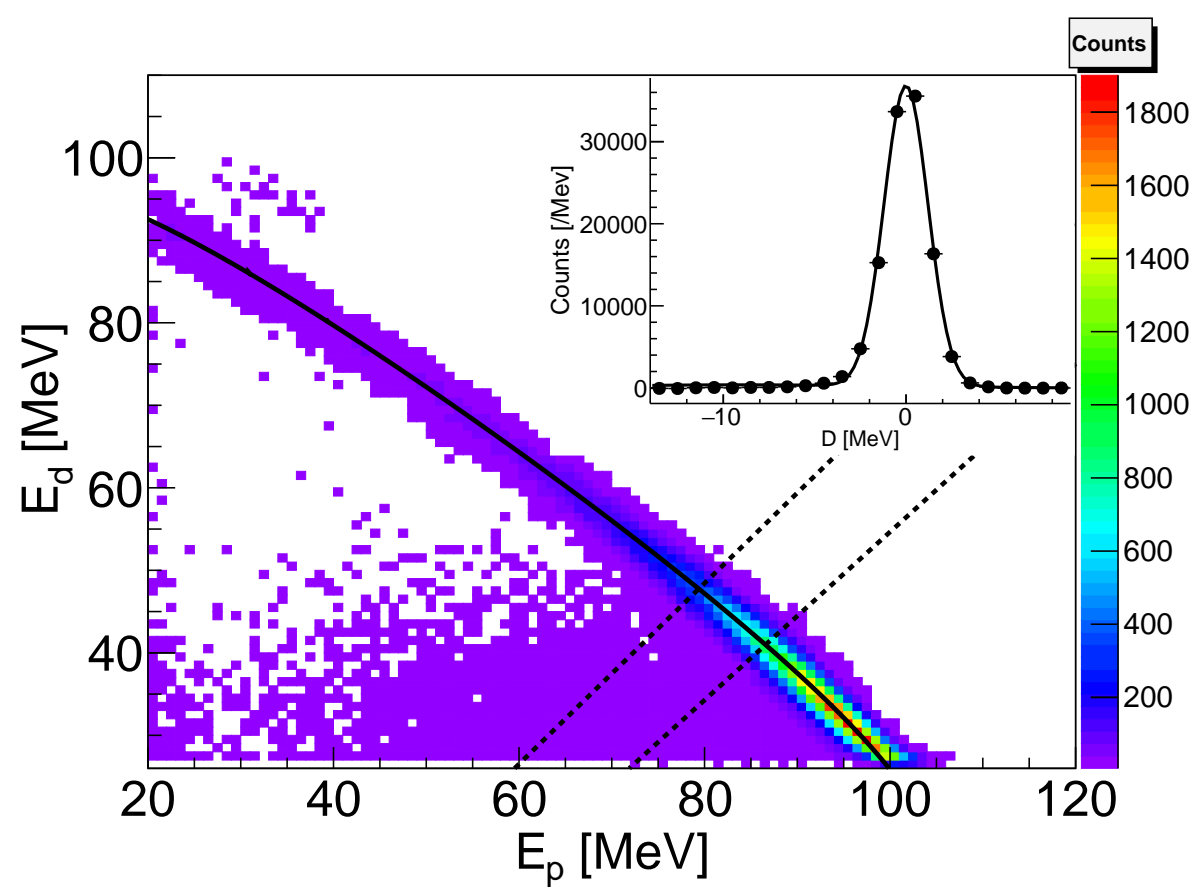

Figure 1: The energy correlation between deuteron and proton for the configuration $\left(\theta_{d}, \theta_{p}, \phi_{12}\right)=\left(25^{\circ} \pm 2,25^{\circ} \pm 2,180^{\circ} \pm 5\right)$ after particle identification. The solid line represents the expected kinematical $S$-curve originating from the kinematical calculation. The dashed lines indicate one of the $S$-intervals. The inset figure shows the result of projected events for this $S$-interval on an axis (D) perpendicular to the S-curve.

a polarized beam is defined as

$$
\begin{aligned}
\sigma(\xi, \phi)=\sigma_{0}(\xi)\left[1+\sqrt{3} p_{Z} \operatorname{Re}\left(i T_{11}(\xi)\right)\right. & \cos (\phi) \\
& -\frac{1}{\sqrt{8}} p_{Z Z} T_{20}(\xi)-\frac{\sqrt{3}}{2} p_{Z Z} \operatorname{Re}\left(T_{22}(\xi)\right) \cos (2 \phi),
\end{aligned}
$$

where $\sigma\left(\sigma_{0}\right)$ is the 5 -fold differential cross section of the reaction with polarized (unpolarized) beam and $\xi$ represents the kinematical variables of each configuration, $\left(\theta_{d}, \theta_{p}, \phi_{12}, S\right)$. $p_{Z}$ and $p_{Z Z}$ are the vector and tensor polarizations, respectively. $\operatorname{Re}\left(i T_{11}\right)$ and $\left(\operatorname{Re}\left(T_{20}\right)\right.$, and $\operatorname{Re}\left(T_{22}\right)$ ) are vector (tensor) analyzing powers and $\phi$ is azimuthal scattering angle of the deuteron. Using data obtained from a pure vector polarized beam, $\left(p_{Z Z}=0\right)$, the $\operatorname{Re}\left(i T_{11}\right)$ is extracted from the amplitude of the $\cos \phi$-shape of the fit function of Eq. 1. Data extracted from a pure tensor polarized beam, $\left(p_{Z}=0\right)$, produces a $\cos 2 \phi$-shape of the azimuthal asymmetry with an offset from one due to the term, $\frac{1}{\sqrt{8}} p_{z Z} T_{20}(\xi)$, in Eq. 1 . The amplitude of the $\cos 2 \phi$-shape yields $\operatorname{Re}\left(T_{22}\right)$ and the offset from one gives $\operatorname{Re}\left(T_{20}\right)$.

\section{Experimental results and discussion}

Via a dedicated analysis of vector and tensor analyzing powers of the ${ }^{2} \mathrm{H}(\vec{d}, d p) n$ break-up reaction [37], we are interested in identifying the quasi-free domain with the neutron as the spectator in the ${ }^{2} \mathrm{H}(\vec{d}, d p) n_{\text {spec }}$ reaction. To proceed, the reconstructed momentum distribu- 
tion of the missing neutron for different intervals of the Mandelstam variable, $u$, is compared with the expected momentum distribution of the nucleon derived from the wave function of the deuteron; see Fig. 4 of Ref. [37]. The regions where the reconstructed momentum distribution of the missing neutron matches well with the expected momentum distribution of the neutron inside the deuteron are labeled as the quasi-free regions. A detailed discussion on the quasi-free domain can be found in [37].

Figure 2 depicts the results of analyzing powers as a function of $S$ for four coplanar configurations including data corresponding to the QF region. Two energy calibration methods have been used to extract the observables. The average has been taken by using the results of two calibration procedures. The differences $(2 \sigma)$ are indicated by a gray band for each configuration. The scattering angle of the deuteron is fixed to be $30^{\circ} \pm 1$ and that for the proton varies between $25^{\circ} \pm 1$ to $35^{\circ} \pm 1$ for each configuration. These results are compared with the results of recent theoretical calculations based on SSA by using CD-Bonn $+\Delta$ potential, applicable for coplanar configurations. In SSA, the break-up amplitude is calculated using the first term in the Neumann series expansion of the corresponding exact four-nucleon equations. We expect this single-scattering approximation to provide a rough estimation of three-body breakup observables in quasi-free kinematics. The single-scattering break-up amplitude consists of 4 terms two terms of which come from the break-up of the deuteron target and the other two terms come from their counterparts, namely, the break-up of the incident deuteron. In SSA1, just the first term of deuteron-target break-up in which neutron acts as a spectator is included while in SSA4, all 4 terms are included $[29,30]$. The red (black) lines correspond to calculations based on SSA1 (SSA4). The blue dashed line indicates the energy of outgoing neutron in the unit of MeV. The energy of the neutron is assumed to be less than $0.3 \mathrm{MeV}$ in the QF region. In other words, the QF region is around the peak in the SSA1 and SSA4 calculations. In some of the configurations, the SSA1 and SSA4 predictions are very close to each other indicating that the SSA1 term is dominant in those cases.

Figure 2 shows that the calculations based on SSA underestimate the measured vector analyzing power $\left(\operatorname{Re}\left(i T_{11}\right)\right)$ for configurations with small proton-deuteron opening angles while the same model agrees with data for larger opening angles. This might point to a deficiency related to the Coulomb barrier. Also, the regions in which the SSA1 and SSA4 predictions are very close to each other, the agreement bwetween data and the SSA calculations is very good. Comparing the results of tensor analyzing power $\left(\operatorname{Re}\left(T_{20}\right)\right)$ indicates a general agreement between SSA and the data especially for the configurations in which the scattering angle of the proton is less than $30^{\circ}$. The data and the results of the calculations for $\operatorname{Re}\left(T_{22}\right)$ agree well with each other for almost all configurations. As a general conclusion, our data show that the single-scattering approximation provides a reasonable estimate of the analyzing powers in the QF region. We do note, however, that significant discrepancies can be observed which are not surprising considering validity of the approach for the low energy considered in this work.

In summary, in a dedicated analysis of the break-up reaction in the $d+d$ system, it has been shown that in identifying regions of kinematics for the study of the QF reaction, one should not only constrain the momentum of the spectator neutron to low values, but also consider the momentum transfer between the beam projectile and the ejectile in the analysis. Our data show that at small momentum transfer, the effects of final-state interactions involving the neutron play an important role. A detailed four-body calculation is required to digest the underlying reaction dynamics that appear at this part of the phase space. The results of the analyzing powers are compared with the single-scattering approximation for the coplanar configurations that generally agree well within the uncertainties. Together with the upcoming state-of-the-art $a b$-initio calculations, these data will provide further insights into the dynamics of three- and four-nucleon forces in few-nucleon systems. 


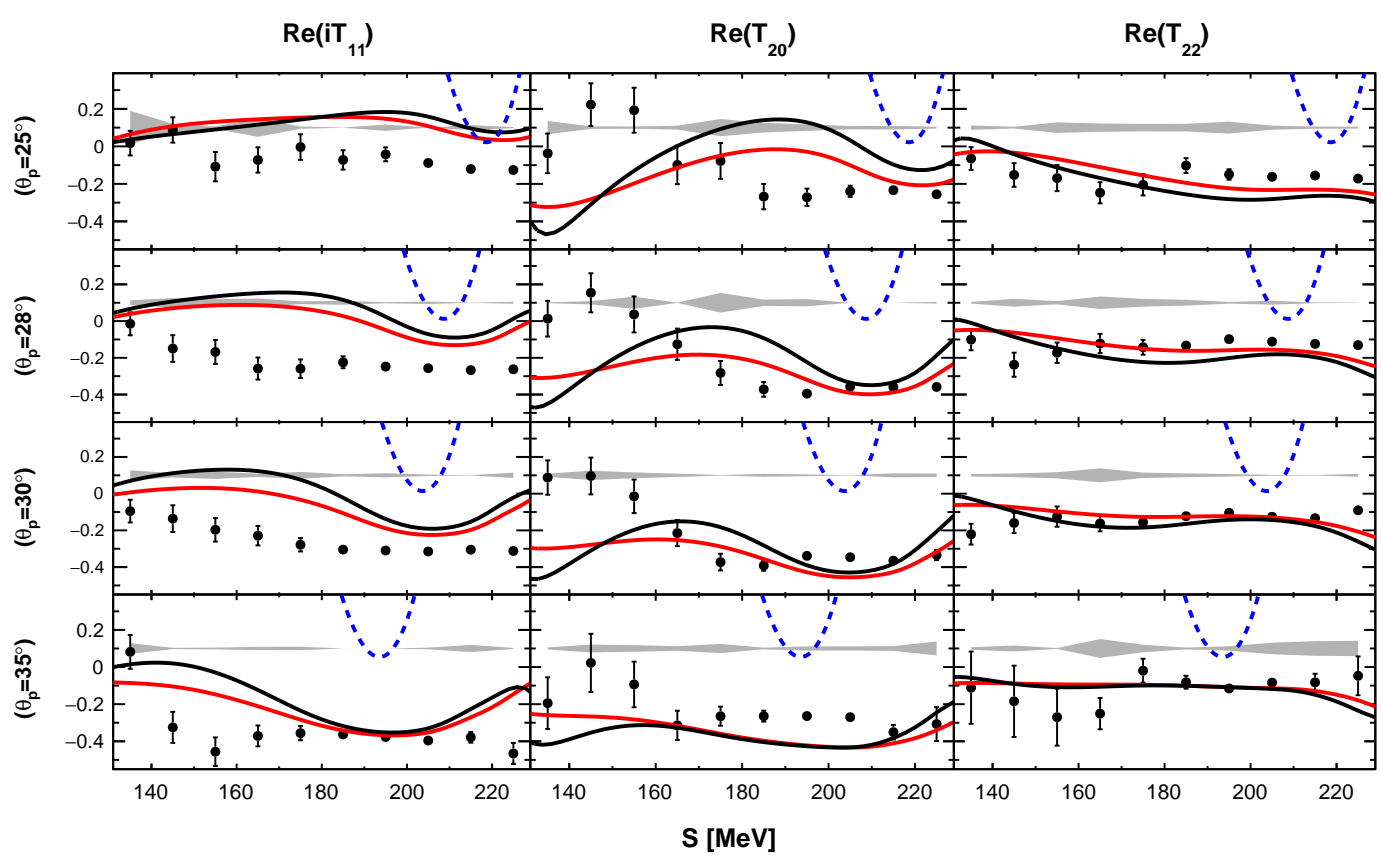

Figure 2: The vector and tensor analyzing powers for three-body break-up reaction presented as a function of $S$ for four coplanar configurations including QF regions. The deuterons are scattered toward $30^{\circ} \pm 1$ and the scattering angles of the protons are presented on the left side. The red (black) lines show the results of the calculations based on SSA1 (SSA4). The blue dashed line indicates the energy of outgoing neutron in the unit of MeV. The gray bands show systematic uncertainty due to the calibration procedure.

\section{References}

[1] H. Yukawa, linearen Atomkette, Proc. Phys. Math. Soc. Jpn. 17, 48 (1935), doi:10.1007\%2FBF01341708.

[2] R. B. Wiringa, R. A. Smith, and T. L. Ainsworth, Nucleon-nucleon potentials with and without $\Delta$ (1232) degrees of freedom, Phys. Rev. C 29, 1207 (1984), doi:https://doi.org/10.1103/PhysRevC.29.1207.

[3] S. Pieper, V. Pandharipande, and R. Wiringa, Realistic models of pion-exchange three-nucleon interactions, Phys. Rev. C 64, 1 (2001), doi:https://doi.org/10.1103/PhysRevC.64.014001.

[4] K. Ermisch et al., Systematic investigation of three-nucleon force effects in elastic scattering of polarized protons from deuterons at intermediate energies, Phys. Rev. C 71, 064004 (2005), doi:https://doi.org/10.1103/PhysRevC.71.064004.

[5] H. Sakai et al., Precise Measurement of $d p$ Elastic Scattering at 270 $\mathrm{MeV}$ and Three-Nucleon Force Effects, Phys. Rev. Lett. 84, 5288 (2000), doi:https://doi.org/10.1103/PhysRevLett.84.5288.

[6] K. Sekiguchi et al., Resolving the Discrepancy of $135 \mathrm{MeV}$ pd Elastic Scattering Cross Sections and Relativistic Effects, Phys. Rev. Lett. 95, 162301 (2005), doi:https://doi.org/10.1103/PhysRevLett.95.162301. 
[7] H. Amir-Ahmadi et al., Three-nucleon force effects in cross section and spin observables of elastic deuteron-proton scattering at $90 \mathrm{MeV} /$ nucleon, Phys. Rev. C 75, 041001(R) (2007), doi:https://doi.org/10.1103/PhysRevC.75.041001.

[8] P. Mermod et al., Evidence of three-body force effects in neutrondeuteron scattering at $95 \mathrm{MeV}$, Phys. Rev. C 72, 061001(R) (2005), doi:https://doi.org/10.1103/PhysRevC.72.061002.

[9] H. Mardanpour et al., Precision measurement of vector and tensor analyzing powers in elastic deuteron-proton scattering, Eur. Phys. J. A 31, 383 (2007), doi:https://doi.org/10.1140/epja/i2006-10228-0.

[10] E. Stephan et al., Vector and tensor analyzing powers of elastic deuteron-proton scattering at $130 \mathrm{MeV}$ deuteron beam energy, Phys. Rev. C 76, 057001 (2007), doi:https://doi.org/10.1103/PhysRevC.76.057001.

[11] E. J. Stephenson et al., Indications of three-nucleon force effects in the proton analyzing power for 70-200 MeV p+d elastic scattering, Phys. Rev. C 60, 061001 (1999), doi:https://doi.org/10.1103/PhysRevC.60.061001.

[12] A. Ramazani-Moghaddam-Arani et al., Elastic proton-deuteron scattering at intermediate energies, Phys. Rev. C 78, 014006 (2008), doi:https://doi.org/10.1103/PhysRevC.78.014006.

[13] St. Kistryn et al., Evidence of the Coulomb-force effects in the cross-sections of the deuteron-proton breakup at $130 \mathrm{MeV}$, Phys. Lett. B 641, 23 (2006), doi:https://doi.org/10.1016/j.physletb.2006.08.013.

[14] N. Kalantar-Nayestanaki et al., A Small-Angle Large-Acceptance Detection System for Hadrons, Nucl. Instrum. and Meth. Phys. Res. A 444, 591 (2000), doi:10.1016/S01689002(99)01179-1.

[15] N. Kalantar-Nayestanaki et al., Signatures of three-nucleon interactions in few-nucleon systems, Rep. Prog. Phys. 75, 016301 (2012), doi:10.1088/0034-4885/75/1/016301.

[16] H. Mardanpour et al., Spin-isospin selectivity in three-nucleon forces, Phys. Lett. B 687, 149 (2010), doi:10.1016/j.physletb.2010.03.021.

[17] E. Stephan et al., Vector and tensor analyzing powers in deuteronproton break-up at $130 \mathrm{MeV}$, Phys. Rev. C 82, 014003 (2010), doi:https://doi.org/10.1103/PhysRevC.82.014003.

[18] R. Ramazani-Sharifabadi et al., High precision data on elastic dd scattering at 65 $\mathrm{MeV} /$ nucleon, IL NUOVO CIMENTO 42 C 129 (2019), doi:10.1393/ncc/i2019-191293.

[19] B. M. Fisher et al., Proton- ${ }^{3}$ He elastic scattering at low energies, Phys. Rev. C 74, 034001 (2006), doi:https://doi.org/10.1103/PhysRevC.74.034001.

[20] A. M. Micherdzinska et al., Deuteron-deuteron elastic scattering at 231.8-MeV, Phys. Rev. C 75, 054001 (2007), doi:103/PhysRevC.75.054001.

[21] A. Deltuva et al., Neutron- ${ }^{3} \mathrm{H}$ scattering above the four-nucleon breakup threshold, Phys. Rev. C 86, 011001(R) (2012), doi:https://doi.org/10.1103/PhysRevC.86.011001. 
[22] A. Deltuva et al., Calculation of proton- ${ }^{3} \mathrm{He}$ elastic scattering between 7 and $35 \mathrm{MeV}$, Phys. Rev. C 87, 054002(R) (2013), doi:https://doi.org/10.1103/PhysRevC.87.054002.

[23] A. Deltuva et al., Calculation of Multichannel Reactions in the Four-Nucleon System above Breakup Threshold, Phys. Rev. Lett. 113, 102502 (2014), doi:https://doi.org/10.1103/PhysRevLett.113.102502.

[24] A. Deltuva et al., Calculation of neutron ${ }^{3} \mathrm{He}$ scattering up to $30 \mathrm{MeV}$, Phys. Rev. C 90, 044002 (2014), doi:https://doi.org/10.1103/PhysRevC.90.044002.

[25] R. Lazauskas, Modern nuclear force predictions for $n-{ }^{3} \mathrm{H}$ scattering above the three- and four-nucleon breakup thresholds, Phys. Rev. C 91, 041001(R) (2015), doi:https://doi.org/10.1103/PhysRevC.91.041001.

[26] A. Deltuva et al., Proton- ${ }^{3} \mathrm{H}$ scattering calculation: Elastic and charge-exchange, Phys. Rev. C 91, 034001 (2015), doi:https://doi.org/10.1103/PhysRevC.91.034001.

[27] A. Deltuva et al., Deuteron-deuteron scattering above four-nucleon breakup threshold, Phys. Lett. B 742, 285-289 (2015), doi:https://doi.org/10.1016/j.physletb.2015.01.052.

[28] A. Deltuva et al., Four-body calculation of ${ }^{2} \mathrm{H}(d, p)^{3} \mathrm{H}$ and ${ }^{2} \mathrm{H}(d, n)^{3} \mathrm{He}$ reactions above breakup threshold, Phys. Rev. C 9, 024003 (2017), doi:https://doi.org/10.1103/PhysRevC.95.024003.

[29] A. Deltuva et al., Four-body calculation of elastic deuteron-deuteron scattering, Phys. Rev. C 92, 024001 (2015), doi:https://doi.org/10.1103/PhysRevC.92.024001.

[30] A. Deltuva et al., Three-cluster break-up in deuteron-deuteron collisions: Single-scattering approximation, Phys. Rev. C 93, 044001 (2016), doi:https://doi.org/10.1103/PhysRevC.93.044001.

[31] H. R. Kremers et al., Polarized Gas Targets and Polarized Beams, AIP Conj Proc. 421, p. 507 (1997).

[32] N. Kalantar-Nayestanaki, J. Mulder, and J. Zijlstra, Thin synthetic windows for cryogenic targets, Nucl. Instrum. and Meth. in Phys. Res. A 417, 215 (1998), doi:https://doi.org/10.1016/S0168-9002(98)00758-X.

[33] H. Mardanpour, Investigation of nuclear forces in $d+p$ elastic and $p+d$ break-up reactions at intermediate energies, Ph.D. thesis, University of Groningen, (2008).

[34] A. Ramazani-Moghaddam-Arani, Cross-section and analyzing-power measurements in three and four-nucleon scattering, Ph.D. thesis, University of Groningen, (2009).

[35] H. R. Kremers et al., The KVI Lamb-shift polarimeter, Nucl. Instrum. and Meth. in Phys. Res. A 516, 209 (2004), doi:https://doi.org/10.1016/j.nima.2003.07.002.

[36] A. Ramazani-Moghaddam-Arani et al., Three-body break-up in deuterondeuteron scattering at $65 \mathrm{MeV} /$ nucleon, Phys. Rev. C 83, 024004 (2011), doi:10.1103/PhysRevC.83.024002.

[37] R. Ramazani-Sharifabadi et al., Investigation of the quasi-free domain in deuteron-deuteron break-up using spin observables, Eur. Phys. J. A 55, 177 (2019), doi:10.1140/epja/i201912876-1.

[38] S. A. Coon et al., Reworking the Tucson-Melbourne Three-Nucleon Potential, Few-Body Syst 30, 131 (2001), doi:https://doi.org/10.1007/s006010170022. 
[39] A. Ramazani-Moghaddam-Arani et al., Spin observables in the three-body break-up process near the quasi-free limit in deuteron-deuteron scattering, Phys. Lett. B 725, 282-286 (2013), doi:10.1016/j.physletb.2013.07.041. 